\title{
FT \\ Creación de un club de escritores a favor de la composición de textos escritos
}

\author{
Creation of a writers club in favor of the composition of written texts
}

Recibido febrero 2019

Arbitrado marzo 2019

Publicado mayo 2019
Egilda Guerra

egildaguerra@hotmail.com

Código ORCID: 0000-0003-3090-0360

Escuela Básica Privada "San Vicente de Paúl”, Venezuela

\section{Resumen}

La presente investigación tuvo como objetivo promover la composición de textos escritos a través de un club de escritores dirigido a niños de Educación Primaria. Para lograrlo se emplearon las metodologías cuantitativa y cualitativa y el diseño fue un proyecto especial, fundamentado en una investigación de campo de carácter descriptivo e interpretativo. La muestra estuvo conformada por niños de tercer grado de la Escuela Básica Privada "San Vicente de Paúl", ubicada en Guatire, estado Miranda, Venezuela. Los resultados arrojaron que los docentes no utilizan estrategias novedosas y creativas en la orientación de la composición escrita y que las estrategias diseñadas para la creación del club de escritores se adaptaron a los intereses y motivaciones de los niños. En fin, esto permitiría que los niños al componer textos escritos reflejaran sus emociones, sus pensamientos y sus sentimientos de forma clara, libre y creativa, favoreciendo el aprendizaje significativo.

\footnotetext{
Abstract

The objective of this research was to promote the composition of written texts through a writers club aimed at children in Primary Education. To achieve this, quantitative and qualitative methodologies were used and the research design was a special project, based on descriptive and interpretive field research. The sample was made up of third grade children from the Private Basic School "San Vicente de Paúl", located in Guatire, Miranda state, Venezuela. The results showed that teachers do not use innovative and creative strategies in the orientation of written composition and that the strategies designed for the creation of the writers club were adapted to the interests and motivations of the children. In short, this would allow children to compose written texts to reflect their emotions, their thoughts and their feelings in a clear, free and creative way, favoring meaningful learning.
}

Palabras clave:

Estrategias instruccionales; composición de textos escritos; promoción de la escritura; club de escritores

\section{Keywords:}

Instructional strategies; composition of written texts; promotion of writing; writers club 


\section{INTRODUCCIÓN}

$\mathrm{U}$ no de los objetivos primordiales de la Educación Primaria, señalados en el Currículo Básica Nacional, es lograr que los niños consoliden su lectura y su escritura (Ministerio de Educación, 1997). No obstante, en algunas instituciones educativas de Venezuela la situación pareciera ser diferente, ya que el aprendizaje de la lengua escrita está caracterizado por ser un proceso rígido solo guiado por el docente $\mathrm{y}$ apegado estrictamente a los aspectos formales de la escritura.

La realidad en el sistema educativo venezolano muestra algunas dificultades relacionadas con la manera como el docente aborda estrategias para la enseñanza de la lectura y de la escritura; así lo demuestran los trabajos realizados por Odremán (2005) quien señala que esta situación se genera por la manera como el docente orienta el aprendizaje de la escritura, el cual está sujeto solo a la dimensión gramatical, desconociendo la percepción de cada estudiante en el acto de escribir; asimismo, se observa que el estudiante recibe sanciones por parte del docente cuando se desarrolla el proceso de escribir, de forma natural y espontánea. Estas sanciones ocurren cuando el docente no acepta textos producidos por los estudiantes de manera original y creativa, pues exige que se escriba lo que él indica.

Los trabajos de esta autora demuestran los graves problemas que presentan los estudiantes de los diferentes niveles educativos al momento de redactar textos escritos y cómo el dominio de esta habilidad se relaciona con el éxito en la vida escolar del estudiante.

Existen investigaciones como la de Russo (2009) y D'amato (2010) donde se evidencia que con la promoción de la lectura se ha intentado solucionar esta situación; sin embargo, es necesario que la promoción de la lectura vaya acompañada por el fomento de la composición de textos escritos para lograr eficientes lectores y escritores desde una visión humana y sensible donde las emociones y los sentimientos sean elementos determinantes.

Morles (1999) señala:

El Ministerio de Educación ha realizado durante décadas, esfuerzos por revisar y actualizar los programas educativos, pero los docentes en general, por fuerza de costumbre siguen la práctica tradicional y 
continúan usando enfoques basados en el desarrollo de habilidades específicas. (p. 70)

Las habilidades específicas de las que habla el autor se refieren a la enseñanza de la ortografía, la acentuación, los signos de puntuación y la sintaxis o estructuras oracionales como elementos formales de la lengua escrita; lo que quiere decir que el docente dirige su enseñanza hacia un enfoque basado en la gramática. Lo anterior trae como consecuencia que se obvia el enfoque basado en el proceso de composición de textos escritos el cual se interesa por los aspectos cognoscitivos de la producción textual; por ejemplo, cómo se generan, relacionan y organizan las ideas en el texto. Bajo este último enfoque, la actividad del aula está más dirigida al proceso de la elaboración textual que al producto final (Sánchez, 1993).

De lo anterior, se deduce que la dinámica escolar y el poco conocimiento del enfoque basado en el proceso de composición de la escritura en las aulas hacen que los docentes realicen prácticas escolares aburridas que no favorecen el desarrollo y adecuado uso de la lengua en todas sus modalidades: hablar, escuchar, leer y escribir.

La situación antes descrita ha originado que los estudiantes perciban el acto de escribir solo como una reproducción de ideas extraídas y copiadas de un texto o de un pizarrón; esto dificulta el adecuado dominio de la lengua escrita desde una perspectiva funcional, humanística y estética.

Tradicionalmente, las actividades de escritura han sido relegadas a la escuela y algunos docentes se limitan a desarrollar solo los aspectos gramaticales y formales como se señaló anteriormente, obviando la esencia del arte de escribir; es decir, escribir desde la funcionalidad del lenguaje (Cassany, 1990); lo anterior no cumple con las exigencias curriculares descritas anteriormente en cuanto a la consolidación de la escritura.

La falta de interés del niño hacia la producción de textos escritos es algo que tanto el docente responsable como padres y representantes deben resolver. En este sentido, se debe fomentar la producción de textos escritos de forma libre sin restricciones o parámetros rígidos y normativos; es decir, ofrecer al niño la posibilidad de escribir desde su visión, sus intereses y sus inquietudes con el propósito de que se active la escritura libre y creativa (Serrano de Moreno, 2002). 
Para lograr esta meta, es necesario que el docente propicie situaciones de aprendizaje que sean significativas y diseñe estrategias que estimulen la composición escrita desde esta visión. Las prácticas pedagógicas recomendadas son aquellas que conciben a la escritura funcional como un proceso, las cuales han sido desarrolladas fundamentalmente por la ciencia cognitiva. Autores como Smith (1982), Bereiter y Scardamalia (1992), Camps (2003), Flower y Hayes (1980), entre otros, se han preocupado por describir y explicar el proceso de la escritura y para ello se han apoyado en los desarrollos de la lingüística, la psicolingüística y la ciencia cognitiva. Dado que estos modelos se centran en el desarrollo de los procesos cognitivos y sociales del escritor, ellos ofrecen opciones nuevas de trabajo en el aula. Estos modelos proponen al docente componer los textos escritos cumpliendo con tres fases: la planificación, la elaboración de los borradores y la revisión.

Desde esta visión, la escuela es una institución social sobre la cual recae la responsabilidad de enseñar a leer y a escribir y donde, por lo general, se asocia el hecho de escribir con los actos de copiar del pizarrón, hacer copias de libros o realizar caligrafías; todo esto se ha evidenciado durante largo tiempo. Situación que debería ser motivo de reflexión de docentes, especialistas del área e instituciones responsables en la implementación y desarrollo de los programas educativos en el país.

Es frecuente que algunos docentes solo orienten prácticas con actividades tradicionales tales como: la copia, la caligrafía, el dictado, entre otras sin brindar al estudiante la oportunidad de disfrutar ese proceso desde su percepción y sus experiencias. En realidad, el manejo de la copia y la caligrafía se asocian a actividades pasivas con la intencionalidad de controlar la atención o la disciplina dentro del aula. No obstante, el uso de la copia y la caligrafía puede ser provechoso, si el docente desarrolla actividades creativas a partir de ellas, las cuales generarían aprendizajes significativos en sus alumnos (Flores, 2008).

Por ello, la presente investigación propuso la creación de un club de escritores para estudiantes del 3er. grado de Educación Primaria; para generar diferentes estrategias a favor de la composición de textos escritos, con el fin de producir un cambio de actitud en el docente y, por ende, en las estrategias de enseñanza, ya que en la promoción de la escritura el papel del docente como mediador es fundamental. De allí que la presente investigación se planteara los siguientes objetivos: 


\section{Objetivo general}

Promover la composición de textos escritos a través de un club de escritores dirigido a los niños cursantes del tercer grado del nivel de Educación Primaria en la Escuela Básica Privada "San Vicente de Paúl", ubicada en Guatire - estado Miranda, Venezuela.

\section{Objetivos específicos}

1. Diagnosticar las estrategias empleadas por los docentes de tercer grado del nivel de Educación Primaria en la Escuela Básica Privada "San Vicente de Paúl" para orientar la composición de textos escritos en sus estudiantes.

2. Diseñar estrategias instruccionales que se pongan en práctica a través de un club de escritores, para promover la composición de textos escritos en los estudiantes de tercer grado del nivel de Educación Primaria en la Escuela Básica Privada "San Vicente de Paúl".

\section{Estrategias instruccionales}

Para Dorrego y García (1991) las estrategias instruccionales son parte importante dentro del diseño instruccional, ya que estas comprenden el conjunto de eventos, actividades, técnicas y medios instruccionales dirigidos a lograr los objetivos del aprendizaje.

Según Szczurek (1989):

La estrategia instruccional es un conjunto de acciones deliberadas y arreglos organizacionales para llevar a cabo la situación de enseñanza-aprendizaje. Comprende la consideración de los siguientes componentes: técnicas instruccionales, actividades, organización de la secuencia, organización de grupos, organización del tiempo y organización del ambiente. (p. 7)

En esta investigación, se asume la definición dada por Szczurek sobre estrategia instruccional por considerarla como una serie de arreglos organizados con un fin didáctico; en la que se incluyen todos los elementos necesarios para ejecución. Para este autor, los componentes fundamentales de un proceso de instrucción son los siguientes: elementos directrices, estudiantes, docentes, medios y otros recursos, evaluación y estrategias.

Elementos directrices. Son los que orientan globalmente el proceso, indicando lo que se espera lograr. Generalmente esos 
logros se expresan en términos de lo que el estudiante aprenderá como consecuencia de la instrucción. Pueden aparecer bajo uno o más de los siguientes nombres o formas: fines, propósitos, metas, contenidos, objetivos (generales, terminales, específicos, expresivos, etc.). Los elementos directrices constituyen el "qué" o "para qué" del proceso de instrucción.

Estudiantes. Protagonistas del proceso de instrucción, traen sus propias características como individuo y como grupos. Dichas características no se refieren solo a los previos conocimientos, habilidades, destrezas y actitudes, sino que también deben considerarse las comúnmente englobadas bajo el término de "características biopsicosociales". El componente estudiantes constituye parte del "quién" o "con quién" del proceso de instrucción.

El docente. Se trata de un componente importante del proceso y, sin embargo, no suele considerarse como tal en los modelos de diseño de instrucción. Posiblemente se deba a que las características de los docentes como individuos, como grupo y en la interacción con los estudiantes, se han estudiado aún menos que las características de los propios estudiantes. El docente constituye la otra arte del "quién" o "con quién" del proceso.

Medios y otros recursos. Se entiende por medio cualquier persona, organismo u objeto que proporciona la información necesaria para facilitar un determinado aprendizaje de conocimientos, actitudes o habilidades (Szczurek, 1989). Algunos medios pueden requerir de otros recursos adicionales para que se efectúe la transmisión de la información; por ejemplo, una película requiere de un artefacto llamado proyector, así como un objeto real microscópico requiere del microscopio. Así mismo, son recursos los materiales tales como aparatos, instrumentos, equipos, herramientas, útiles y sustancias. Los medios y otros recursos como componentes del proceso de instrucción constituyen el "con qué".

Evaluación. Se debe considerar tanto la evaluación de los aprendizajes como la evaluación del diseño mismo formativa y sumativamente. El componente de evaluación constituye el "qué tan bien" del proceso de instrucción. 
Estrategia. La estrategia instruccional constituye el "cómo" del proceso instruccional. La estrategia no debe tratarse de forma aislada, sino en el contexto de sus interrelaciones con los otros elementos del diseño instruccional. La estrategia como elemento del sistema instruccional debe considerarse a su vez como un sistema con sus propios elementos, interrelaciones y propósito.

La estrategia constituye entonces una de las piezas fundamentales de los procesos de enseñanza y aprendizaje, puesto que de ella dependen la orientación y operatividad del proceso e implica una interrelación con los demás elementos del diseño instruccional, como lo son: los objetivos, los contenidos, las conductas de entrada de los alumnos, los medios instruccionales y la evaluación.

Asumiendo que las estrategias instruccionales comprenden un conjunto de acciones, se toman los planteamientos de Alfonzo (2003) quien señala que se dividen en tres eventos y momentos específicos: estrategias de inicio, de desarrollo y de cierre, donde cada una cumple con una función específica en el proceso de enseñanza. Así se tiene que: a) las estrategias de inicio son para activar la atención, establecer el propósito, incrementar el interés, la motivación y recordar conocimientos previos; b) las estrategias de desarrollo permiten conceptuar el contenido, procesar la nueva información, utilizar estrategias de aprendizaje y practicar y c) las estrategias de cierre sirven para revisar y resumir la clase, transferir el aprendizaje, proponer enlaces y cerrar la clase.

Por otra parte, también existen los momentos y eventos instruccionales que, según Alfonzo (2003), son los pilares guías para las estrategias instruccionales, considerando que estarán dirigida a una audiencia de educación primaria y al tipo de contenidos u objetivos del currículo de este nivel. Al respecto, este autor plantea lo siguiente:

Momento del inicio: en esta fase se prepara al estudiante para la instrucción, se activa la atención y se promueven la motivación y el interés. Los cuatro eventos instruccionales señalados para el momento de inicio son los siguientes: activar la atención, establecer el propósito, incrementar el interés y la motivación y presentar la visión preliminar de la lección.

Momento del desarrollo: en este se presenta y procesa la nueva información. 
Momento de cierre: tiene el propósito de revisar el aprendizaje logrado para utilizarlo en diferentes contextos y abrir la posibilidad de adquirir o construir nuevos aprendizajes y de establecer enlaces con otros contenidos.

Lo anteriormente describe los eventos instruccionales que forman parte de la estrategia instruccional propuesta y constituye una guía sistemática para la planificación de acciones educativas, no obstante, en la práctica pedagógica algunos de ellos se omiten o se unen.

\section{El proceso de composición escrita}

La concepción de la escritura ha logrado en la actualidad un cambio significativo, generado por los resultados de varias investigaciones que han permitido reconocerla como un proceso de expresión del pensamiento, de expresión de manera de pensar y de comunicación de forma escrita.

Según Serrano (2002) escribir es la capacidad del hombre para expresar sus pensamientos, ideas, sentimientos y necesidades por medio de la palabra escrita. La enseñanza de la lengua se dirige hacia el desarrollo del individuo que involucra el logro de competencias que le permitan comunicarse con su entorno en diferentes situaciones.

Para fines de la presente investigación, la enseñanza se orienta al desarrollo de la competencia comunicativa, para Cassany et al. (2000) la competencia comunicativa es la capacidad de usar el lenguaje apropiadamente en diversas situaciones sociales de comunicación.

Desde esta perspectiva, el papel de la escuela es preponderante, ya que como institución tiene la responsabilidad de formar individuos que dominen el lenguaje y adquieran las herramientas para saber qué, cómo, cuándo, a quién y dónde expresar de forma escrita sus pensamientos y emociones.

En el proceso de escritura, se observan varios sub- procesos que están estrechamente relacionados. Ellos son: la planificación o preparación, la producción del escrito o elaboración de borradores, la revisión y la edición definitiva.

En la planificación se recauda el material, se lee, se selecciona y se desecha aquello que no guarda relación con lo que se quiere expresar, también se determina el propósito de lo que se quiere comunicar y a quién va dirigido. 
En la elaboración de borradores quién escribe tiene la oportunidad de interactuar con el texto, ubicarse desde la óptica de otro punto de vista. Asimismo puede agregar o eliminar una palabra.

La revisión permite a quien escribe visualizar el texto elaborado y los conceptos emitidos, descubrir si el contenido de lo que escribe tiene coherencia y sentido para quien lee; esta actividad requiere de esfuerzo y tiempo por parte del docente, quien debe procurar que el alumno asuma un rol crítico sobre lo que escribe. La revisión puede realizarse en forma individual o grupal siempre con el apoyo del docente.

Una vez realizada la revisión se procede a la edición definitiva del texto, es el momento para la revisión general, para luego proceder a redactar el texto definitivo.

Es necesario que el docente asuma la enseñanza de la lectura y escritura desde una perspectiva crítica y que sea modelo de sus alumnos como auténticos lectores y escritores. Para ello, el docente debe formarse constantemente a través de talleres, cursos, seminarios, entre otros, relacionados con la enseñanza de la lengua escrita.

\section{La promoción de la escritura. El club de escritores}

En los últimos años, la idea que se tenía acerca de la escritura como un proceso mecánico y apegado a reglas y normas ha cambiado; la nueva visión valora este proceso como una forma de expresar el pensamiento, las ideas y los sentimientos que van a permitir, entre otros, comunicarse.

Para lograr formar niños como escritores autónomos, es necesario que dentro de las aulas de clases se promocione el desarrollo de estrategias instruccionales que motiven a los niños a escribir lo que piensan, lo que sienten y dar a conocer lo que saben.

Lo antes expuesto permite determinar los fines de esta investigación, que pretende promover la escritura a partir de la creación de un club de escritores, un espacio dedicado a desarrollar el arte de escribir y donde se considere la escritura como un proceso activo de construcción, en el cual el sujeto que aprende tiene un papel protagónico que le permite construir textos en función de sus actitudes, aptitudes, habilidades y conocimientos que ya posee.

Es importante señalar que para promover la escritura es necesario considerar los aspectos que relaciona la lengua oral con 
la lengua escrita, en ambas se considera la comprensión y la producción; en la primera se logra a través de lo que escucha y lo que lee y, en la segunda, a través de lo que habla y escribe.

Entonces para promover la escritura es necesario abordar la promoción de la lectura. A través de ella, el niño entra en contacto con el texto, disfruta de este y también le permite desarrollar su pensamiento crítico y conectarse con sus emociones, sentimientos e inquietudes.

Desde esta visión, se puede considerar la lengua escrita como una conexión entre la lectura y la escritura, que permite la formación afectiva y social del sujeto que aprende. En la intención de destacar el valor que tiene ese contacto entre los niños y el texto desde una perspectiva diferente a la ya adoptada, la literatura juega un rol determinante, debido a que cuenta con valiosos recursos como los cuentos, las adivinanzas, las retahílas, los poemas y canciones, entre otros. Estos textos literarios podrían ser utilizados por el docente para acercar al niño a la lectura y aumentar ese contacto para que luego los niños aprecien su valor desde una visión estética.

Tanto la escuela y el hogar deben brindar situaciones y experiencias a los niños que les permitan apropiarse de esta herramienta para comunicarse por escrito, expresar sus sentimientos y sus inquietudes a través de una expresión caracterizada por la creatividad.

La literatura representa y debe ser considerada un rol fundamental en la escuela y en el hogar; su valor artístico permite descubrir en el niño la capacidad de crear, aumentar su sensibilidad, fortalecer valores y disfrutar su momento para leer. Por lo tanto, se hace necesario acercar a los niños al deleite, disfrute y vivencia de los textos literarios, a través de una interacción entre docente y alumnos, promoviendo espacios para que les permitan vivir sus aventuras imaginativas y que sean la lectura y escritura un vehículo para lograrlo. Para ello se puede promover la escritura a partir de un club de escritores.

$\mathrm{El}$ club de escritores nace como una propuesta innovadora que pretende promover la escritura a través de la creación de un espacio que brinde a los alumnos la oportunidad de disfrutar de la lectura de diversos materiales impresos para luego componer textos significativos; también sería un espacio para conversar y dialogar temas de interés.

En la actualidad existen algunas definiciones sobre club de lectura escolar como la conceptualización dada por Peña y 
Bardoza (como se citó en Peña, 2009), cuando manifiestan: "El Club de Lectura Escolar es una organización alrededor de la cual se agrupan padres, representantes, alumnos y docentes con la finalidad de propiciar la lectura desde una visión estética, donde los sentimientos y las emociones sean permitido aflorar" (p. 1).

En cuanto a la conceptualización de club de escritores, no se encontraron evidencias; por tanto, se define como un grupo de personas que se reúnen para escribir textos significativos, para luego comentarlo entre sus integrantes. Así mismo se considera que los textos significativos son todos aquellos textos que transmiten un mensaje preciso, con claridad y coherencia; además deben tener una funcionalidad, sentido o propósito claramente definido por el escritor. En ellos se pueden expresar sentimientos, alegrías, tristezas de acuerdo con la situación que se esté viviendo. Están dirigidos a destinatarios previamente seleccionados, tales como amigos, compañeros y familiares. Estos textos son fuentes de imaginación, creación, posibilidad de conocimientos, expresión, desarrollo del pensamiento y reflexión sobre lo que pasa en la vida del ser humano.

El club de escritores pretende propiciar un espacio de entretenimiento a través de la escritura de textos significativos y fomentar la participación activa y efectiva de cada integrante para promover la producción escrita de forma individual y grupal. El club puede funcionar como un grupo abierto y democrático donde todos los integrantes tienen las mismas responsabilidades.

¿Quiénes integran el club de escritores? Puede estar integrado por cualquier tipo de personas a las que les interese escribir de forma natural y espontánea; en este caso, alumnos de la institución.

¿Qué ventajas ofrece el club de escritores? La escritura tiene un valor decisivo en la formación del individuo, en la estructuración de su pensamiento y en su actuación dentro de la sociedad a la que pertenece. Hoy nos encontramos sumergidos en la llamada "sociedad de la información", la cual exige el dominio de ciertas habilidades. Por lo tanto, los ciudadanos tienen la necesidad de adaptarse a estas exigencias y así evitar ser excluidos. Entonces, el club de escritores ofrece un espacio para promover la creatividad y el dominio de la lengua hablada y la lengua escrita.

Importancia del club de escritores. La implementación de un club de escritores en un espacio educativo permite fomentar la 
escritura y lectura de diversos textos literarios, para ampliar el conocimiento, como también sensibilizar a los escritores y lectores. Se convierte así en un lugar para fomentar el diálogo y el respeto por las opiniones ajenas y donde la escritura abre sus espacios desde una visión diferente.

Objetivos educacionales. Entre los objetivos relacionados con la escritura están: a) promover la producción escrita en los niños de tercer grado de educación primaria para mejorar su proceso de lecto-escritura; b) proporcionar orientaciones didácticas para favorecer el aprendizaje significativo en pro de la formación de escritores críticos, autónomos, analíticos y participativos; c) orientar el proceso de la composición escrita en niños de tercer grado de educación primaria.

Objetivos del club de escritores. Entre los objetivos del club de escritores se pueden nombrar los siguientes: a) ofrecer a los niños situaciones de contacto libre con diversos materiales; b) propiciar un espacio dedicado a la interacción con la escritura; c) incitar a los niños a leer todos los días; d) respetar el ritmo de aprendizaje de cada alumno, tomando en cuenta que el arte de escribir es un proceso que requiere de tiempo y dedicación por parte del docente.

\section{MÉTODO}

L a presente investigación fue producto de un estudio mayor. Estuvo enmarcada dentro de los paradigmas cuantitativo y cualitativo, a su vez, fundamentada en un proyecto especial y una investigación de campo de carácter descriptivo.

Siguiendo los planteamientos de Cook y Reichardt (1995) acerca de la necesidad de la integración de los paradigmas cuantitativo y cualitativo, esta investigación asumió el enfoque integrado entre la metodología cualitativa y cuantitativa para atender mejor las exigencias del problema de la estudio, cuyo propósito no puede ser alcanzado por un solo paradigma; proceso que tiene que ver con la formulación de propuestas, creación de teorías y modelos a través de la combinación de métodos cualitativos y cuantitativos.

Por su parte, Bericat (como se citó en La Torre et al., 1996) señala que en la investigación se puede hacer uso de lo que él llama estrategia de complementación; refiere que esta se produce 
cuando en el marco de una misma investigación se obtienen dos imágenes: una procedente de métodos de orientación cuantitativa y otras de métodos de orientación cualitativa, donde cada perspectiva ilumina una dimensión diferente de la realidad que se investiga; de manera que no existe solapamiento entre los métodos.

En este estudio se recogieron los datos para el diagnóstico de manera cuantitativa y, durante la aplicación de la estrategia, se recopiló información cualitativa sobre los intereses y apreciaciones de los sujetos de la investigación.

En cuanto al diseño, el presente trabajo de investigación se insertó dentro de la modalidad de proyecto especial fundamentado en una investigación de campo. El proyecto especial se refiere a:

Trabajos que lleven a creaciones tangibles, susceptibles de ser utilizadas como soluciones a problemas demostrado, o que respondan a necesidades e intereses de tipo cultural. Se incluyen en esta categoría los trabajos de elaboración de libros de textos y materiales de apoyo educativo, el desarrollo de software educativos, prototipo y de productos tecnológicos en general, así como también de los de creación literaria y artística. (UPEL, 2016, p. 22)

Esta investigación generó un proyecto especial porque se elaboró un material tangible como fue el diseño de un manual que representa un material de apoyo educativo dirigido a los docentes que laboran en los primeros grados del nivel de Educación Primaria.

El diseño de este estudio permitió analizar los hechos y la realidad de institución. En la investigación de campo, los datos obtenidos son denominados datos primarios, porque el investigador trabaja directamente en el contexto seleccionado y con los sujetos de dicho contexto.

El nivel de la investigación se suscribe a un nivel descriptivo. Según Hernández et al. (2004), los estudios descriptivos se centran en medir con la mayor precisión posible y que el investigador debe ser capaz de definir qué se va a medir y cómo lograr con precisión esa medición; así como también requiere de un considerable conocimiento del área que se investiga.

La población de una investigación en palabras de Palella y Martins (2004) se refiere al "conjunto de unidades de las que se desea obtener información y sobre las que se van a generar 
conclusiones" (p. 92). En esta investigación, la población estuvo representada por todos los estudiantes y tres docentes que laboran en las tres secciones de tercer grado administrados en la E. B. P. "San Vicente de Paúl”; dos de esas secciones funcionan en el turno de la mañana y otra en el turno de la tarde.

En este mismo orden de ideas, la muestra seleccionada se hizo atendiendo al concepto que de ella hacen Palella y Martins (2004), quienes la definen como "la escogencia de una parte representativa de una población, la muestra representa un subconjunto de la población, accesible y limitado, sobre el que realizamos las mediciones" (p. 94).

La selección de la muestra fue intencional y los criterios fueron establecidos por la investigadora: del turno de la mañana y la sección con menos estudiantes. Es así que la muestra seleccionada para llevar a cabo la presente investigación se constituyó en 22 estudiantes del tercer grado, sección $\mathrm{B}$ y los tres docentes que administran dicho grado en esta institución.

A objeto de establecer el contacto con la realidad en estudio, se utilizó la técnica de la observación, que definida por Sabino (1999), se tiene como el uso sistemático de nuestros sentidos orientados a la captación de la realidad que queremos observar.

Esta técnica se adaptó perfectamente a este trabajo de investigación, ya que al abordar la escritura desde una perspectiva grupal se facilitó ampliamente la labor de la investigadora, al dar margen adecuado para intervenir y recabar toda la información requerida sin que el grupo a estudiar percibiera algún tipo de intervención más allá de la contemplada en los lineamientos de la propuesta.

En este estudio, se emplearon como instrumentos de recolección de datos una lista de cotejo para el diagnóstico inicial, y un cuaderno de registro para la aplicación de las estrategias diseñadas.

El procedimiento utilizado se organizó en dos etapas.

\section{Etapa I. Diagnóstico de las estrategias empleadas por los docentes}

En esta etapa, la investigadora observó el desarrollo de la clase en dos momentos para cada sección (terceros A, B y C). En total, se efectuaron seis observaciones para determinar las estrategias que empleaban los docentes, que administran estas secciones, para orientar la composición de textos escritos en sus estudiantes. 
Para ello, se diseñó una lista de cotejo, la cual estuvo conformada por 14 ítems con tres alternativas: totalmente presentes, totalmente ausentes y parcialmente ausentes. Este instrumento fue validado por tres expertos.

Los datos reportados en este instrumento constituyeron la base para el diseño de las estrategias instruccionales, considerando las debilidades observadas durante el desarrollo de las clases.

\section{Etapa II. Diseño del club de escritores}

El diseño del club de escritores se efectuó en tres fases:

Fase I. Se realizó una revisión bibliográfica a fin de determinar los lineamientos para la conformación de un club.

Fase II. Se procedió al diseño de las estrategias instruccionales a través de un club de escritores, para promover la composición de textos escritos en los estudiantes de Tercer Grado del Nivel de Educación Primaria de la Escuela Básica Privada "San Vicente de Paúl". En total, se diseñaron diez estrategias instruccionales.

Fase III. En esta fase se diseñó un instrumento para validar las estrategias diseñadas y se entregó a dos expertos en Lengua y Literatura y un experto en estrategias de aprendizaje.

\section{RESULTADOS}

\section{Etapa I. Diagnóstico de las estrategias empleadas por los docentes}

En esta etapa, se identificaron las estrategias empleadas por los docentes de tercer grado de Educación Primaria de la Escuela Básica Privada "San Vicente de Paúl" a fin de orientar la composición de textos escritos en sus estudiantes. Para ello, la investigadora asistió a dos clases por cada docente y observó el desarrollo de estas. En total se observaron seis clases. La observación se registró en la lista de cotejo previamente mencionada.

Para la descripción de los datos, se asignó un código a cada docente de la siguiente manera: D.A (docente de 3er Grado A), D.B (docente de 3er Grado B), D.C (docente de 3er Grado C). 
En la Tabla 1, se observa cuáles fueron los indicadores totalmente presentes (en los tres docentes), totalmente ausentes (en los tres docentes) y parcialmente ausentes (en al menos un docente).

Tabla 1. Estrategias instruccionales observadas

\begin{tabular}{|c|c|c|}
\hline ÍTEM & INDICADORES & RANGO \\
\hline 1 & Muestra el objetivo a los estudiantes & $\begin{array}{l}\text { D.A Ausente } \\
\text { D.B Ausente } \\
\text { D.C Ausente }\end{array}$ \\
\hline 2 & Presenta el contenido a aprender & $\begin{array}{l}\text { D.A Presente } \\
\text { D.B Presente } \\
\text { D.C Presente }\end{array}$ \\
\hline 3 & Activa conocimientos previos & $\begin{array}{l}\text { D.A Ausente } \\
\text { D.B Presente } \\
\text { D.C Presente }\end{array}$ \\
\hline 4 & Interactúa con los estudiantes & $\begin{array}{l}\text { D.A Ausente } \\
\text { D.B Presente } \\
\text { D.C Ausente }\end{array}$ \\
\hline 5 & Emplea útiles escolares & $\begin{array}{l}\text { D.A Presente } \\
\text { D.B Presente } \\
\text { D.C Ausente }\end{array}$ \\
\hline 6 & Emplea materiales y equipos & $\begin{array}{l}\text { D.A Ausente } \\
\text { D.B Ausente } \\
\text { D.C Ausente }\end{array}$ \\
\hline 7 & Desarrolla la exposición oral & $\begin{array}{l}\text { D.A Ausente } \\
\text { D.B Ausente } \\
\text { D.C Ausente }\end{array}$ \\
\hline 8 & $\begin{array}{l}\text { Promueve la interacción docente - } \\
\text { estudiante }\end{array}$ & $\begin{array}{l}\text { D.A Ausente } \\
\text { D.B Ausente } \\
\text { D.C Ausente }\end{array}$ \\
\hline 9 & Define los elementos directrices & $\begin{array}{l}\text { D.A Presente } \\
\text { D.B Presente } \\
\text { D.C Ausente }\end{array}$ \\
\hline 10 & $\begin{array}{l}\text { Organiza la clase de manera lógico- } \\
\text { jerárquica }\end{array}$ & $\begin{array}{l}\text { D.A Ausente } \\
\text { D.B Ausente } \\
\text { D.C Ausente }\end{array}$ \\
\hline 11 & Trabaja con pequeños grupos & $\begin{array}{l}\text { D.A Ausente } \\
\text { D.B Ausente } \\
\text { D.C Presente }\end{array}$ \\
\hline 12 & Trabaja en forma individual & $\begin{array}{l}\text { D.A Presente } \\
\text { D.B Presente } \\
\text { D.C Ausente }\end{array}$ \\
\hline
\end{tabular}




\begin{tabular}{cll}
\hline ÍTEM & INDICADORES & \multicolumn{1}{c}{ RANGO } \\
\hline \multirow{2}{*}{13} & \multirow{2}{*}{ Evalúa los aprendizajes } & D.A Presente \\
& & D.B Presente \\
& & D.C Presente \\
14 & Evalúa todo el proceso didáctico & D.A Ausente \\
& & D.B Presente \\
& & D.C Ausente \\
\hline
\end{tabular}

Los indicadores totalmente presentes son: a) presenta el contenido a aprender, y b) evalúa los aprendizajes. Estos son dos aspectos que siempre están en todas las clases, los docentes se dedican a impartir contenidos programáticos, donde el control de información solo es manejado por el docente, el estudiante está sujeto a las decisiones y pautas del maestro $\mathrm{y}$, al final de la actividad, es evaluado para verificar qué tanto aprendió.

Los indicadores totalmente ausentes son: a) Muestra el objetivo a los estudiantes; b) emplea materiales y equipos; c) desarrolla la exposición oral, y d) promueve la discusión dirigida. En la práctica se evidenció que al trabajar con los niños, pareciera que el docente considera que no es importante explicarles el objetivo o propósito de la actividad del día; en la exposición oral del docente no se observa la presentación de la nueva información de forma clara y precisa. La práctica está asociada a lo que exige el docente; en cuanto a las representaciones gráficas tampoco se evidenció su manejo, asimismo en el resumen de la lección. No se observó el empleo de materiales novedosos y atractivos propios del nivel de escolaridad.

Los indicadores parcialmente ausentes son: a) Activa conocimientos previos; b) Interactúa con los estudiantes; c) Emplea útiles escolares; d) define los elementos directrices; e) trabaja con pequeños grupos; f) Trabaja en forma individual, y g) evalúa todo el proceso didáctico. El docente presta muy poca atención a indagar inicialmente qué tanto saben sus estudiantes sobre el tema que se abordará. Las preguntas intercaladas se evidenciaron solo en una oportunidad, esto indica que no lo lleva a una interacción constante en el aula para intercambiar ideas y opiniones.

En todas las clases observadas, se evidenció que los docentes exigían a los niños hacer uso de la lengua escrita, es decir, indistintamente del tema o asignatura a abordar, los estudiantes 
debían producir algún texto escrito. Sin embargo, en ninguna de las clases, las docentes observadas orientaron el proceso de composición escrita con una estrategia completamente ajustada a los elementos directrices y al proceso de escritura tal como lo establecen autores como Szczurek (1989) y Casanny (1990), respectivamente. En fin, las estrategias manejadas por los docentes en la Escuela Básica Privada "San Vicente de Paúl" relacionadas con la promoción de textos escritos se presentan de forma poco novedosa y creativa. De allí surgió la necesidad de diseñar y proponer, en esta investigación, estrategias instruccionales que permitieran cambiar la visión del docente en el momento de orientar la composición de textos escritos en sus estudiantes.

Una vez diagnosticadas las estrategias que empleaba el docente de tercer grado en esta institución se procedió a diseñar el club de escritores.

\section{Etapa II: Diseño del club de escritores}

Esta etapa se ejecutó en tres fases: en la primera (revisión bibliográfica) se destinó a considerar en profundidad los basamentos que servirían de marco teórico a la presente investigación, las teorías seleccionadas fueron: el aprendizaje significativo de Ausubel (1976), la teoría de Jean Piaget (1975) y el enfoque sociocultural de Vigotsky $(1976,1977)$; en la segunda fase (diseño de las estrategias) se presentaron diez estrategias con la siguiente estructura: nombre de la estrategia, organización de la secuencia, contenidos a trabajar, objetivos, características de los estudiantes, características del docente, momentos, eventos, medios y recursos, técnicas, organización del ambiente, organización del grupo, organización del tiempo y la evaluación.

Después de diseñadas las estrategias, se elaboró un material instruccional (Guerra, 2014), cuya presentación contiene una atractiva diagramación y su estructura consta de las siguientes partes: una portada, un índice, una introducción, un apartado dedicado a la fundamentación teórica, presentación de los íconos a utilizar, seguidamente la descripción de cada estrategia seleccionada (nombre de la estrategia, organización de la secuencia, objetivo, contenidos, características de los docentes, características de los estudiantes, momentos: inicio, desarrollo y cierre, eventos, medios y recursos, técnicas, organización), anexos y, por último, las referencias bibliográficas. En el tercer momento 
de esta etapa, las estrategias diseñadas fueron validadas por dos expertos en el área de Lengua y Literatura y otro en estrategias.

En la Tabla 2 se observa información sintetizada de las estrategias que fueron desarrolladas.

Tabla 1. Nombre de la estrategia instruccional, objetivo y contenido

\begin{tabular}{|c|c|c|}
\hline Nombre de la estrategia & Objetivo & Contenido \\
\hline $\begin{array}{l}\text { Estrategia } \mathbf{N}^{\circ} 1 \\
\text { CREACIÓN DEL CLUB DE } \\
\text { ESCRITORES (Primera parte) }\end{array}$ & $\begin{array}{l}\text { Conocer la } \\
\text { funcionalidad del club } \\
\text { de escritores. }\end{array}$ & $\begin{array}{l}\text { Definición de club, } \\
\text { sus normas y } \\
\text { reglamentos }\end{array}$ \\
\hline $\begin{array}{l}\text { Estrategia } N^{\circ} 2 \\
\text { CREACIÓN DEL CLUB DE } \\
\text { ESCRITORES (Segunda } \\
\text { parte) }\end{array}$ & $\begin{array}{l}\text { Constituir el club de } \\
\text { escritores. }\end{array}$ & $\begin{array}{l}\text { Nombre del club, } \\
\text { horario y } \\
\text { responsabilidades }\end{array}$ \\
\hline $\begin{array}{l}\text { Estrategia } \mathrm{N}^{\circ} 3 \\
\text { ¿CÓMO ESCRIBIR } \\
\text { CORRECTAMENTE? }\end{array}$ & $\begin{array}{l}\text { Analizar las } \\
\text { características de un } \\
\text { buen escritor. }\end{array}$ & $\begin{array}{l}\text { Concepto de } \\
\text { escritura, importancia } \\
\text { del buen escribir }\end{array}$ \\
\hline $\begin{array}{l}\text { Estrategia } \mathrm{N}^{\circ} 4 \\
\text { ¿CÓMO CONVENCER } \\
\text { ESCRIBIENDO? }\end{array}$ & $\begin{array}{l}\text { Desarrollar en los } \\
\text { alumnos la capacidad } \\
\text { de argumentar por } \\
\text { escrito. }\end{array}$ & $\begin{array}{l}\text { La argumentación y } \\
\text { su estructura }\end{array}$ \\
\hline MIS NOTAS PERSONALES & $\begin{array}{l}\text { Descubrir la función } \\
\text { social y comunicativa } \\
\text { del lenguaje escrito. }\end{array}$ & $\begin{array}{l}\text { Funciones de la } \\
\text { lengua escrita }\end{array}$ \\
\hline $\begin{array}{l}\text { DIALOGUEMOS CON LA } \\
\text { NOTICIA }\end{array}$ & $\begin{array}{l}\text { Promover el diálogo } \\
\text { como medio para } \\
\text { intercambiar } \\
\text { experiencias y } \\
\text { opiniones. }\end{array}$ & El diálogo \\
\hline $\begin{array}{l}\text { Estrategia } \mathbf{N}^{\circ} 7 \\
\text { NOTICIA FANTÁSTICA }\end{array}$ & $\begin{array}{l}\text { Despertar el interés } \\
\text { por la lectura de la } \\
\text { prensa y activar la } \\
\text { fantasía a través de } \\
\text { textos escritos. }\end{array}$ & $\begin{array}{l}\text { La noticia, } \\
\text { características y } \\
\text { estructura }\end{array}$ \\
\hline $\begin{array}{l}\text { Estrategia } \mathrm{N}^{\circ} 8 \\
\text { ANAGRAMA DE PALABRAS }\end{array}$ & $\begin{array}{l}\text { Proponer a los } \\
\text { estudiantes juego de } \\
\text { palabras que les } \\
\text { permita examinar su } \\
\text { estructura y } \\
\text { composición. }\end{array}$ & $\begin{array}{l}\text { Formación de } \\
\text { palabras }\end{array}$ \\
\hline PAREJA DISPAREJA & $\begin{array}{l}\text { Crear historias breves } \\
\text { vinculando dos } \\
\text { palabras que no tienen } \\
\text { relación. }\end{array}$ & El sustantivo \\
\hline $\begin{array}{l}\text { Estrategia } \mathbf{N}^{\circ} 10 \\
\text { ESCUCHA Y DESCUBRE }\end{array}$ & $\begin{array}{l}\text { Lograr que los } \\
\text { estudiantes reconozcan } \\
\text { y den forma lúdica al } \\
\text { lenguaje, a través de la } \\
\text { adivinanza. }\end{array}$ & $\begin{array}{l}\text { La adivinanza y sus } \\
\text { características }\end{array}$ \\
\hline
\end{tabular}




\section{CONCLUSIONES}

T as estrategias instruccionales permiten un aprendizaje significativo ya que logran una intensa actividad mental de reflexión en cuanto a: qué, cómo, por qué y para quién escribir, también se observó un cambio positivo por parte de los estudiantes en el momento de componer textos escritos.

La orientación por parte del docente en la composición de textos escritos implica el diseño de estrategias que ayuden, impulsen y motiven un comportamiento escritor por parte del docente y los estudiantes. Esta actividad debe ser totalmente placentera para los niños, debe permitirles a ellos expresar lo que piensan, sienten y lo que conocen. Dar a conocer sus emociones y opiniones. Para ello, la práctica de la composición de textos escritos debe ser frecuente en el aula, formar parte de la cotidianidad escolar. Es necesario abrir espacios en los que los niños escriban diversos textos, esto permitirá desarrollar su propio lenguaje literario.

\section{REFERENCIAS}

Alfonzo, A. (2003). Estrategias Instruccionales. Trabajo no Publicado. Caracas. Recuperado de http://files.estrategias2010.webnode.es/20000004161b8e62b27/estrategias\%20instruccionales\%20alfonso.pdf Ausubel, D. (1976). Psicología Educativa. México: Trillas Bereiter, C. y Scardamalia, M. (1992). Dos modelos explicativos de los procesos de composición escrita. Revista Infancia $y$ Aprendizaje. 58, 50 -60

Cassany, D. (1990). Enfoque Didáctico para la Enseñanza de la Expresión Escrita: La comunicación de Lenguaje y Educación. Madrid: Aprendizaje

Cassany, D., Luna, M. y Sanz, G. (2000). Enseñar Lengua. Barcelona: Graó

Camps, A. (2003). Miradas diversas a la enseñanza y el aprendizaje de la composición escrita. Revista Lectura y Vida. 4 (24), 2-11

Cook, T. y Reichardt, Ch. (1995). Métodos cualitativos y cuantitativos en investigación educativa. Madrid: Morata

D’amato, M. C. (2010). Estrategias instruccionales basadas en el aprendizaje estratégico para promover el proceso de adquisición de la lectura en niños de primer grado del Grupo Escolar Guatire (trabajo de grado). Instituto Pedagógico de Miranda José Manuel Siso Martínez, Universidad Pedagógica Experimental Libertador (UPEL), La Urbina 
Dorrego, C. y García, G. (1991). Dos modelos para la producción y la evaluación de materiales instruccionales. Caracas: Fondo editorial Facultad de Humanidades y Educación U.C.V

Flores, C. (2008). El aprendizaje estratégico como medio para abordar la composición escrita en estudiantes de educación superior (tesis doctoral). Instituto Pedagógico de Caracas, Universidad Pedagógica Experimental Libertador (UPEL), Caracas

Flower, L. y Hayes, J. (1980). The dynamics of composing: marking plans and juggling constraints. Cognitive Processes in Writing. Hillsdale. New Jersey: Lawrence Earlbaum Associates

Guerra, E. (2014). Estrategias instruccionales dirigidas a través de un club de escritores para la composición de textos escritos en alumnos de tercer grado en la Escuela Básica Privada "San Vicente de Paúl" Guatire-estado Miranda (trabajo de grado). Instituto Pedagógico de Miranda José Manuel Siso Martínez, Universidad Pedagógica Experimental Libertador, Caracas, Venezuela

Hernández. R., Fernández C., C. y Baptista L., P. (2004). Metodología de la investigación. México: McGraw-Hill. Interamericana

La Torre, C. Del Rincón, D. y Arnal, J. (1996). Investigación cualitativa en educación. Barcelona: Gráfique

Ministerio de Educación. (1997). Currículo Básico Nacional. Caracas: Autor

Morles, A. (1999). Los libros de textos y enseñanza de la lectura en Venezuela. Revista de Investigación y Postgrado, 3 -10

Odreman T, N. (2005). Estrategias para el desarrollo de las competencias comunicativas. Brújula Pedagógica. Caracas: El Nacional

Palella, S. y Martins, F (2004). Metodología de la Investigación Cuantitativa. Caracas: Fondo Editorial de la Universidad Pedagógica Experimental Libertador, FEDUPEL

Peña, J. (2009). Creación de un club de lectura. Una experiencia compartida con padres y representantes. Postgrado de Lectura y Escritura. Facultad de Humanidades y Educación. Universidad de Los Andes

Piaget, J. (1975). Psicología y pedagogía. Barcelona: Ariel

Russo, E. (2009). Estrategias Instruccionales para desarrollar la producción textual en alumnos de Tercer Grado de la Unidad Educativa Municipal Bárbaro Rivas (Trabajo de grado). Instituto Pedagógico de Miranda José Manuel Siso Martínez, Universidad Pedagógica Experimental Libertador (UPEL), La Urbina

Sabino, C. (1999). El proceso de Investigación. Caracas: Panapo

Sánchez de R., I. (1993). Coherencia y órdenes discursivos. En Revista Letras. 50. CILLAB. IPC. UPEL 
Serrano de Moreno, S. (2002). Formación de lectores y escritores autónomos. Orientaciones didácticas. Mérida: Editorial Ex Libris

Smith, F. (1982). Writing and the writer. London: Heinemann Educational Book Co

Szczurek, M. (1989). La estrategia instruccional. En Revista de Investigación y Postgrado. 4 (2). 7-26

Universidad Pedagógica Experimental Libertador. (2016). Manual de Trabajos de Grado de Especialización y Maestría y Tesis Doctorales. Caracas: Autor

Vigotsky, L. S. (1976). El Desarrollo de los procesos psicológicos superiores. Barcelona: Crítica

Vigotsky, L. S. (1977). Pensamiento y lenguaje. Teoría del Desarrollo Cultural de las Funciones Psíquicas. Buenos Aires:

La Pirámide 